\title{
Quantification of Glyphosate and AMPA by HPLC-ICP-MS/MS and HPLC-DAD: A Comparative Study
}

\author{
Emanuella M. Pimenta, ${ }^{a}$ Fabio F. da Silva ${ }^{b}$ Érica S. Barbosa, ${ }^{a}$ Ane P. Cacique, ${ }^{a}$ \\ Douglas L. Cassimiro, ${ }^{a}$ Gevany P. de Pinho ${ }^{a}$ and Flaviano O. Silvério ${ }^{\circledR * a}$ \\ ${ }^{a}$ Instituto de Ciências Agrárias, Universidade Federal de Minas Gerais, \\ 39404-547 Montes Claros-MG, Brazil
}

${ }^{b}$ Agilent Technologies Brasil Ltda., 1142 Alameda Araguaia, 06455-940 Barueri-SP, Brazil

\begin{abstract}
In this work, the direct determination methodology of glyphosate and aminomethylphosphonic acid (AMPA) by high performance liquid chromatography (HPLC) using inductively coupled plasma with triple quadrupole mass spectrometer (ICP-MS/MS) was compared with the conventional method using diode array detector (HPLC-DAD). Both methods were selective, but the chromatographic analysis using ICP-MS/MS as detector was four times faster than DAD. The methodology using ICP-MS/MS as detector was linear from 27 to $218 \mu \mathrm{g} \mathrm{L}^{-1}$, while by the conventional method it was 1 to $8 \mathrm{mg} \mathrm{L}^{-1}$. Detection limits of the glyphosate achieved with the HPLC-ICP-MS/MS and HPLC-DAD methods were 8.2 and $300 \mu \mathrm{g} \mathrm{L}{ }^{-1}$, respectively. The direct determination using ICP-MS/MS as detector showed a lower number of stages in sample preparation because it did not need the derivatization step. Seven real samples were analyzed by HPLC-ICP-MS/MS and the results showed that these analytes were not detected.
\end{abstract}

Keywords: aminomethylphosphonic acid, $\mathrm{N}$-(phosphonomethyl)glycine, speciation, derivatization, detection system

\section{Introduction}

Glyphosate ( $N$-(phosphonomethyl)glycine) is an herbicide of great agronomic expression due to wide applicability and efficiency in controlling weeds in many crops. In addition, it was considered toxicologically and environmentally safe for many years, which resulted in a considerable increase in agricultural applications, becoming the most widely adopted herbicide in the world..$^{1,2}$ However, in recent years, this increase has raised doubts and concerns about its increasing use, which in the long run may lead to undesirable impacts on the environment and human health. In 2015, the International Agency for Research on Cancer (IARC) classified glyphosate as a probable carcinogen to humans. ${ }^{3}$

Therefore, many studies with glyphosate and its main metabolite, aminomethylphosphonic acid (AMPA), have been conducted to investigate and develop simple, sensitive and efficient methods for extraction and detection of these compounds in several matrices such as water, ${ }^{4-7}$ soil, ${ }^{8,9}$ fruits ${ }^{10-12}$ and vegetables. ${ }^{13-15}$ However, chemical

*e-mail: flavianosilverio@ufmg.br properties of glyphosate and AMPA such as high polarity, low solubility in organic solvents, absence of chromophore groups and low volatility make their separation and detection processes laborious. ${ }^{12,13}$

These characteristics make an additional derivatization step necessary to detect these compounds through spectroscopic techniques (conventional methods), which may promote an increase in their selectivity and sensitivity. Derivatization is still considered the main sample preparation disadvantage, since this procedure increases the number of sample preparation steps, requires expensive reagent use, and may cause analyte loss when there is not reaction efficiency. In addition, all these factors may contribute to an increase in standard deviation and process cost. ${ }^{16}$

For toxicological and environmental monitoring, several countries and organizations have established significantly different maximum residue limits (MRLs) for glyphosate in drinking water such as $700 \mu \mathrm{g} \mathrm{L}{ }^{-1}$ by the US EPA, ${ }^{17}$ and less than $0.1 \mu \mathrm{g} \mathrm{L}^{-1}$ by European legislation ${ }^{18}$ for this matrix. Therefore, several analytical methods have been developed to determine glyphosate at concentrations below these limits. 
Gas chromatography (GC) and high performance liquid chromatography (HPLC) have been the most commonly used techniques for glyphosate and AMPA quantification, ${ }^{19}$ and the derivatization step has been necessary in both techniques. In this context, direct determination using inductively coupled plasma with triple quadrupole mass spectrometer (ICP-MS/MS) as a detector for HPLC may be more advantageous, because it does not require the derivatization step. The ICP-MS/MS has been used to determine the heteroatoms instead of compounds, i.e., to determine phosphorus in the glyphosate and AMPA molecules. ${ }^{20}$ Moreover, the use of the MS/MS mode eliminates interferences from the matrix, thereby increasing the technique's sensitivity. ${ }^{21}$

The aim of the present work was to compare two detection systems coupled to HPLC in quantifying glyphosate and AMPA, evaluating analytical performance through selectivity, linearity, limit of detection, analysis and sample preparation time. The evaluated systems were diode array detector HPLC-DAD (conventional method) through the molecular detection of derivatized glyphosate and AMPA, and HPLC-ICP-MS/MS by specific detection of ${ }^{31} \mathrm{P}$ isotope present in these compounds.

\section{Experimental}

\section{Reagents and solutions}

Glyphosate $(99.2 \% \mathrm{~m} / \mathrm{m})$ and aminomethylphosphonic acid $(99.0 \% \mathrm{~m} / \mathrm{m})$ were obtained from Sigma-Aldrich (St. Louis, MO, USA). Glyphosate and aminomethylphosphonic acid solutions were prepared at $500 \mathrm{mg} \mathrm{L}^{-1}$ (stock solution) separately in ultrapure water $(18 \mathrm{M} \Omega \mathrm{cm}$ resistivity) obtained by the Milli-Q system (Merck Millipore, Darmstadt, Germany). A solution for each compound was prepared from stock solution at 25 and $5 \mathrm{mg} \mathrm{L}^{-1}$ in the same solvent (working solution). Next, dilutions were made from these solutions to obtain the analytical curves. All solutions were stored at $4{ }^{\circ} \mathrm{C}$ in the refrigerator in plastic bottles to avoid the complexation of metal ion standards in glass vials. ${ }^{2}$ Also, acetonitrile, methanol, 9-fluorenylmethyl chloroformate (FMOC-Cl), phosphoric acid, acetic acid solution $(49 \% \mathrm{~m} / \mathrm{v})$, tetrabutylammonium hydroxide, and ammonium acetate $(65 \%)$ were purchased from Sigma-Aldrich (St. Louis, MO, USA) and sodium borate from QM (São Paulo, Brazil).

Instrumentations

\section{HPLC-DAD}

The analysis was performed on an Agilent 1290 system (Agilent Technologies, Germany) consisting of a quaternary pump, an autosampler, a degasser and a diode array detector. ZORBAX SB-C8 $(4.6 \times 150 \mathrm{~mm}, 5 \mu \mathrm{m})$ was employed for separating glyphosate and AMPA derivatized with FMOC-Cl. The mobile phase composition was $0.2 \%(\mathrm{v} / \mathrm{v})$ phosphoric acid in water (solvent $\mathrm{A}$ ) and acetonitrile (solvent B) at a flow rate of $1 \mathrm{~mL} \mathrm{~min}^{-1}$. The column compartment temperature was set to $25{ }^{\circ} \mathrm{C}$ and the injection volume was $20 \mu \mathrm{L}$. The program was set for a linear gradient starting from $10 \%$ of solvent B to reach $45 \%$ of the solvent at $18 \mathrm{~min}$. After that time, there was a decrease of solvent B from 45 to $10 \%$ in $2 \mathrm{~min}$, reestablishing the initial conditions for the next run. Detection of FMOC-Cl derivatives of each herbicide was performed at $\lambda=250,260$ and $264 \mathrm{~nm}^{8}$

\section{Derivatization}

Glyphosate and AMPA standards were previously derivatized to provide chromophore groups to the compounds. Aqueous standard solutions (1, 2, 4, 6 and $8 \mathrm{mg} \mathrm{L}^{-1}$ ) were prepared for calibration curves, where $100 \mu \mathrm{L}$ of these solutions were transferred to a derivatization vial ( $0.3 \mathrm{~mL}$ capacity) and then derivatized by adding $100 \mu \mathrm{L}$ of $1.25 \mathrm{mM}$ borate buffer ( $\mathrm{pH}$ 9) followed by $100 \mu \mathrm{L}$ of $10 \mathrm{mM}$ FMOC-Cl reagent, and the reaction was allowed to take place for $30 \mathrm{~min}$ at $25^{\circ} \mathrm{C}$ temperature. ${ }^{22} \mathrm{The}$ derivatized extracts were filtered through a $0.45 \mu \mathrm{m}$ syringe filter and finally was directly injected into the HPLC-DAD.

\section{HPLC-ICP-MS/MS}

The high-performance liquid chromatography module (Model 1260 Infinity Series, Agilent Technologies, Waldbronn, Germany) was equipped with a quaternary pump, autosampler, degasser and thermostatic column compartment.

A ICP-MS/MS model Agilent $8800^{\circledR}$ (Agilent Technologies, Hachioji, Japan) was used, equipped with a 3-channel low pressure peristaltic pump (sample, internal standard and drain) and sample introduction system consisting of a concentric nebulizer, double pass spray chamber surrounded by a temperature control system, a quartz torch with $2.5 \mathrm{~mm}$ diameter injector tube, sampling and skimmer platinum cones with orifices of 1 and $0.4 \mathrm{~mm}$ in diameter, respectively. A solid state $27.12 \mathrm{MHz}$ radio frequency generator with helical coils operating between 500 and 1600 Watts of power, fed with argon $99.998 \%$ was used for plasma sustaining. The $8800^{\circledR}$ has a quadrupole for ion selection that will enter in the third generation octopolar reaction system (Octopole Reaction System $\mathrm{ORS}^{\circledR}$ ) pressurized with $99.999 \%$ helium or $99.999 \% \mathrm{O}_{2}$ to remove the isobaric interferences through discrimination kinetic energy or reaction. After the $\mathrm{ORS}^{\circledR}{ }^{\circledR}$, there is another 
quadrupole mass spectrometer with hyperbolic bars with a dual stage electron multiplier detection system (pulsed and analog) at the end, which covers the mass spectrum between 2-260 Da.

The first quadrupole was adjusted to detect $m / z 31\left({ }^{31} \mathrm{P}\right)$, as this is the mass of the phosphorus isotope $\left({ }^{31} \mathrm{P} 100 \%\right)$. In addition, ICP-MS/MS enables using a second analysis mass in MS/MS mode due the reaction with $\mathrm{O}_{2}$ in the collision and reaction cell (CRC), which is positioned between two quadrupole analyzers and was used for removing isobaric interferents.

The $\mathrm{O}_{2}$ was introduced as reaction gas in the $\mathrm{CRC}$, and the second quadrupole was adjusted to monitor the reaction product $m / z 47\left({ }^{31} \mathrm{P}^{16} \mathrm{O}^{+}\right)$, so that these ions were detected with minimal interference: ${ }^{20}$

${ }^{31} \mathrm{P}^{+}+{ }^{16} \mathrm{O}_{2} \rightarrow{ }^{31} \mathrm{P}^{16} \mathrm{O}^{+}+{ }^{16} \mathrm{O} \quad \Delta \mathrm{H}_{\text {reaction }}=-3.17 \mathrm{eV}$

The analytical conditions were: ZORBAX SB-C8 $(4.6 \times 150 \mathrm{~mm}, 5 \mu \mathrm{m})$, injection volume $50 \mu \mathrm{L}$, mobile phase composed of $50 \mathrm{mM}$ acetic acid/ammonium acetate solution: $5 \mathrm{mM}$ tetrabutylammonium: $1 \%$ methanol with $\mathrm{pH}=4.7$, isocratic elution with $1.0 \mathrm{~mL} \min ^{-1} .{ }^{23}$ The HPLC-ICP-MS/MS analysis conditions are summarized in Table 1.

\section{Analytical curves}

Aqueous standard solutions (5, 10, 20, 30 and $40 \mu \mathrm{g} \mathrm{L} \mathrm{L}^{-1}$ of total phosphorus) were prepared in triplicate for HPLC-ICP-MS/MS analyzes. The analytical curves were obtained by placing the concentration values of glyphosate and AMPA on the abscissa axis and the areas were obtained on the ordinate axes using OriginPro 8 software, ${ }^{24}$ which provided the determination $\left(\mathrm{R}^{2}\right)$, angular (a) and linear (b) coefficients of the analytical curves. Extreme values were investigated and excluded by the Jackknife residual test, with a maximum exclusion of $22.2 \%$ of the original number of results, and the ordinary least squares method (OLSM) was again applied to the data for each exclusion.

The assumptions related to regression residuals and linear model adjustment were verified to validate the OLSM: (i) normality of residuals by the Ryan-Joiner test; (ii) homoscedasticity of residuals by the Brown-Forsythe test; (iii) independence of residuals by the Durbin-Watson test; and (iv) the $F$-test to verify the regression significance and linear model adjustment.

\section{Limit of detection}

The limit of detection (LOD) should be the lowest
Table 1. HPLC-ICP-MS/MS operation conditions

\begin{tabular}{lc}
\hline \multicolumn{2}{c}{ ICP-MS/MS parameters } \\
\hline RF power / W & 1600 \\
Plasma gas flow / $\left(\mathrm{L} \mathrm{min}^{-1}\right)$ & 15 \\
Carrier gas flow / $\left(\mathrm{L} \mathrm{min}^{-1}\right)$ & 1.0 \\
Makeup gas flow / $\left(\mathrm{L} \mathrm{min}^{-1}\right)$ & 2.0 \\
Sample depth / $\mathrm{mm}$ & 3 \\
Nebulizer & concentric \\
Nebulization chamber & double-pass \\
Sampling and skimmer cones & platinum \\
Mass monitored & $\mathrm{Q} 1\left(\mathrm{~m} / \mathrm{z}^{31} \mathrm{P}\right)$ and \\
& $\mathrm{Q} 2\left(\mathrm{~m} / z 7^{31} \mathrm{P}^{16} \mathrm{O}^{+}\right)$ \\
Dwell time / (s per isotope $)$ & 0.1 \\
Reaction gas & $\mathrm{O}_{2}$ \\
Reaction gas flow / $\left(\mathrm{L} \mathrm{min}^{-1}\right)$ & 1.5 \\
\hline
\end{tabular}

\begin{tabular}{lc}
\hline HPLC parameters \\
\hline Separation column & ZORBAX SB-C8 \\
& $(4.6 \times 150 \mathrm{~mm}, 5 \mu \mathrm{m})$
\end{tabular}

Temperature $/{ }^{\circ} \mathrm{C}$

30

Mobile phase composition

buffer: $50 \mathrm{mM}$ acetic acid/ammonium acetate $+5 \mathrm{mM}$ tetrabutylammonium $+1 \%$ methanol, $\mathrm{pH}=4.7$

Flow / $\left(\mathrm{mL} \mathrm{min}^{-1}\right) \quad 1$

Injection volume / $\mu \mathrm{L}$ 50

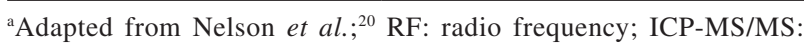
inductively coupled plasma with triple quadrupole mass spectrometer; HPLC: high performance liquid chromatography.

concentration detected in the added samples, but not necessarily quantified. The LOD in this study was obtained by signal-to-noise ratio $(\mathrm{S} / \mathrm{N})$ and determined as three times this relation.

\section{Real sample preparation}

The HPLC-ICP-MS/MS methodology was applied to determine glyphosate and AMPA in water samples from Turmalina city, Minas Gerais, Brazil. This city was chosen for presenting large eucalyptus plantations in its region. We collected water samples from artesian wells, dams, water springs and cisterns. Therefore, these samples had high potential to be contaminated by this herbicide as they were collected near eucalyptus plantation areas. All water samples were collected in $1000 \mathrm{~mL}$ polypropylene bottles. Water aliquots were stored at $4{ }^{\circ} \mathrm{C}$ immediately after sampling. The water samples were filtered through $0.45 \mu \mathrm{m}$ pore sized membrane filters (Millipore, Bedford, MA, USA) before analysis. The manipulation and analysis were developed immediately after sampling. 


\section{Results and Discussion}

We evaluated the analytical performance to compare the two detection systems in the quantification of glyphosate and AMPA through selectivity, analysis time, linearity, limit of detection and sample preparation time.

\section{Selectivity and analysis time}

The chromatogram obtained from determining derivatized glyphosate and AMPA by HPLC-DAD showed several chromatographic signals attributed to by-products of the derivatization reaction with FMOC-Cl. However, the method was considered selective and the compounds eluted in less than $20 \mathrm{~min}$, as shown in Figure 1.

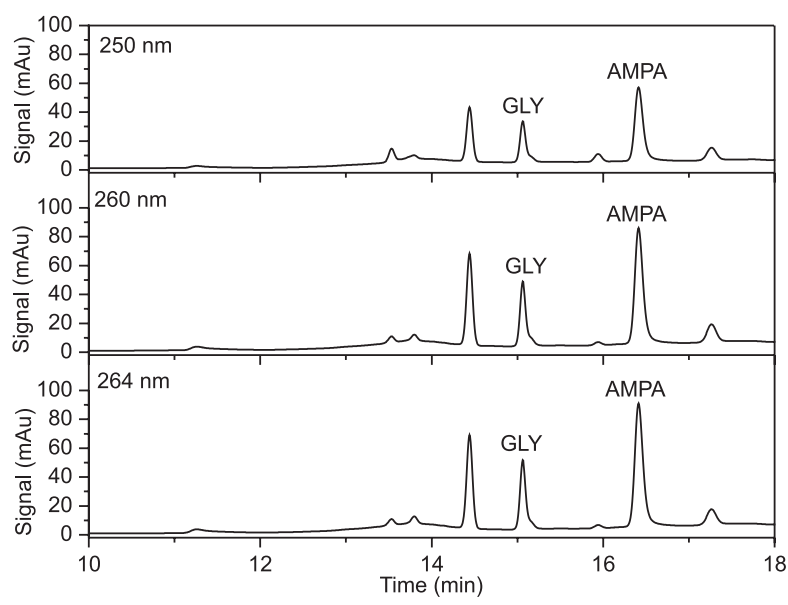

Figure 1. Chromatograms obtained from glyphosate (GLY) and AMPA derivatized standards at $6 \mathrm{mg} \mathrm{L}^{-1}$ by HPLC-DAD.

The main wavelengths used in determining glyphosate and AMPA were 260, ${ }^{25} 240,{ }^{12} 254,206,210$ and $360 \mathrm{~nm}^{26-29}$ The choice of each wavelength was mainly associated to the derivatizing agent used. In this study, the wavelengths 250, 260 and $264 \mathrm{~nm}$ were simultaneously evaluated by comparing the slope of the analytical curves (Table S1, Supplementary Information (SI) section). The angular coefficients in each analytical curves were statistically different when evaluated by the $t$-test $(\alpha=5 \%)$, indicating higher sensitivity of the detector to determine these target analytes in $264 \mathrm{~nm}$, which obtained higher angular coefficient. Therefore, this wavelength was adopted for the comparison analyzes with HPLC-ICP-MS/MS.

The chromatogram obtained from the direct determination of glyphosate and AMPA by HPLC-ICP-MS/MS showed only two chromatographic signals assigned to the two target analytes. This result confirmed the selectivity of the method and the compounds eluted in less than $5 \mathrm{~min}$, as shown in Figure 2.

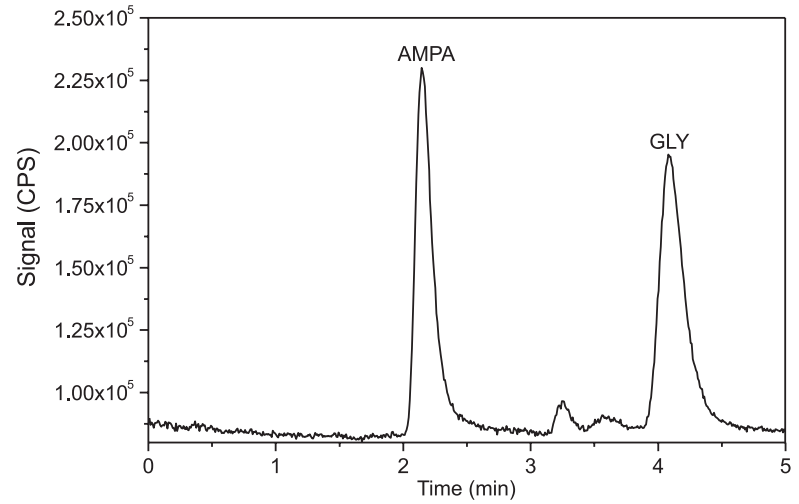

Figure 2. Chromatogram obtained from the direct determination of glyphosate (GLY) and AMPA at the concentration of $50 \mu \mathrm{g} \mathrm{L}^{-1}$ of total phosphorus (corresponding to $273 \mu \mathrm{g} \mathrm{L}^{-1}$ of glyphosate and $179 \mu \mathrm{g} \mathrm{L}^{-1}$ of AMPA) by HPLC-ICP-MS/MS.

In this method, the first quadrupole was programmed to detect $\mathrm{P} m / z=31$ and the second was adjusted to monitor the reaction product ${ }^{31} \mathrm{P}^{16} \mathrm{O}^{+} \mathrm{m} / z=47$. The reaction of ${ }^{31} \mathrm{P}$ with $\mathrm{O}_{2}$ in CRC was explored with the aim to avoid isobaric interferents (polyatomic ions) ${ }^{30}$ such as ${ }^{14} \mathrm{~N}^{1} \mathrm{H}^{16} \mathrm{O}^{+},{ }^{15} \mathrm{~N}^{16} \mathrm{O}^{+}$ and ${ }^{12} \mathrm{C}^{1} \mathrm{H}_{3}{ }^{16} \mathrm{O}^{+}$formed from argon gas plasma, which contribute with the signal intensity of $\mathrm{m} / \mathrm{z}=31 .{ }^{19}$

Different gases such as helium, hydrogen, oxygen and ammonia could be used in CRC, ${ }^{31}$ but $\mathrm{O}_{2}$ was chosen in this study because it was verified in some studies that the reaction with oxygen is thermodynamically favorable and there is a significant increase of sensitivity in detecting phosphorous compounds in food matrices and water. ${ }^{20,23}$ A recent study also obtained better results using oxygen gas for detecting organophosphorus pollutants in water. ${ }^{32}$

The comparison of these analytical parameters showed that both methods were selective, but the method using ICP-MS/MS as the detector presented a four times shorter analysis time and a cleaner chromatogram.

\section{Linearity}

The second analyte parameter evaluated after demonstrating that both methods were selective was linearity. Thus, all outliers were determined and confirmed by the Jackknife residual test. The residual plots and outliers removed for each curve are shown in Figure S1 (SI section). The maximum number of outliers removed was three, corresponding to a limit of $22.2 \%$ of the 15 original data. This step allowed for deleting some points that could have an influence on the fitted regression equation. The results obtained for the tests of normality, homoscedasticity and independency of residuals indicated that the use of OLSM were appropriate. Table S2 (SI section) shows the tabulated critical values and the statistics from the normality, homoscedasticity and independency tests. 
Also, high significance of the regression was observed for all curves (Table 2), indicating adjustment to the linear model at the concentration levels studied for each analyte in both detection systems.

Table 2. Statistics of variance regression analysis for glyphosate and AMPA curves in the investigated detection systems

\begin{tabular}{lccc}
\hline Analyte & Statistic & HPLC-DAD & HPLC-ICP-MS/MS \\
\hline \multirow{2}{*}{ Glyphosate } & $F$ & $1.83 \times 10^{+3}$ & $1.71 \times 10^{+3}$ \\
& $F_{\text {crit }}$ & $1.16 \times 10^{-12}$ & $2.63 \times 10^{-14}$ \\
\hline \multirow{2}{*}{ AMPA } & $F$ & $2.21 \times 10^{+3}$ & $3.57 \times 10^{+3}$ \\
& $F_{\text {crit }}$ & $4.59 \times 10^{-13}$ & $3.17 \times 10^{-16}$ \\
\hline
\end{tabular}

$F$ : statistic test; $F_{\text {crit }}$ : tabulated value. HPLC-DAD: high performance liquid chromatography with diode array detector; HPLC-ICP-MS/MS: HPLC using inductively coupled plasma with triple quadrupole mass spectrometer; AMPA: aminomethylphosphonic acid.

Figure 3 presents the $x-y$ plots and the respective OLSM statistics. These plots demonstrate linearity in the ranges from 1 to $8 \mathrm{mg} \mathrm{L}^{-1}$ for derivatized glyphosate and AMPA by the HPLC-DAD determination, and from 5 to $40 \mu \mathrm{g} \mathrm{L}^{-1}$ of total phosphorus corresponding from 27 to $218 \mu \mathrm{g} \mathrm{L}^{-1}$ of glyphosate and from 18 to $143 \mu \mathrm{g} \mathrm{L}^{-1}$ of AMPA by HPLC-ICP-MS/MS.
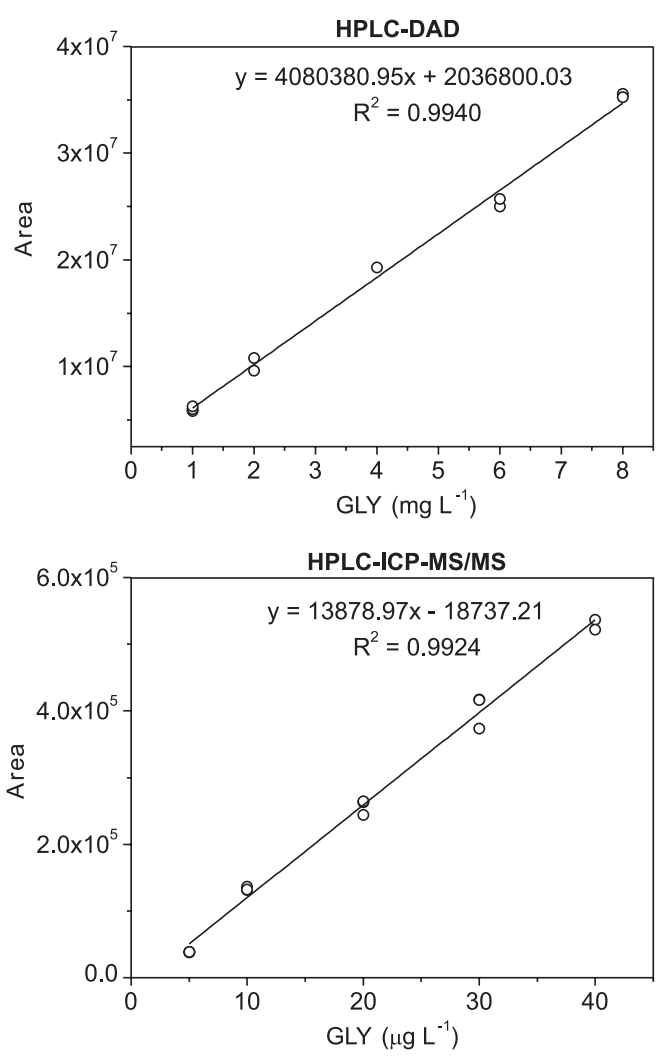

The comparison of these analytical parameters showed that both methods were linear for the two target analytes. However, the methodology using the ICP-MS/MS as detector was linear from 27 to $218 \mu \mathrm{g} \mathrm{L}^{-1}$, while it was 1 to $8 \mathrm{mg} \mathrm{L}^{-1}$ by the conventional method, showing superiority for the HPLC-ICP-MS/MS in relation to HPLC-DAD.

\section{Limit of detection}

In this study, the detection limit achieved for these analytes by HPLC-ICP-MS/MS was 36.5 times lower than HPLC-DAD, as shown in Table 3. These values found in our study were much lower than the MRL established by the US EPA in drinking water $\left(700 \mu \mathrm{g} \mathrm{L}^{-1}\right)$, but higher than that determined by the European Union $\left(0.1 \mu \mathrm{g} \mathrm{\textrm {L } ^ { - 1 }}\right)$.

A recent study using a similar methodology reached a total phosphorous detection limit of 0.02 to $0.69 \mu \mathrm{g} \mathrm{L} \mathrm{L}^{-1}$, corresponding to 0.11 to $3.76 \mu \mathrm{g} \mathrm{L}^{-1}$ of the glyphosate. These values were lower to those achieved in our study. ${ }^{32}$ However, optional gas $\left(1 \% \mathrm{CO}_{2}\right.$ in argon) was used in this study for a carbon enhancement effect of up to 3 -fold in the limit of detection. This effect has generally been used for determination of arsenic, selenium and phosphorous. ${ }^{33}$

Similarly, the detection limit obtained in determining glyphosate and AMPA by HPLC-DAD was higher than
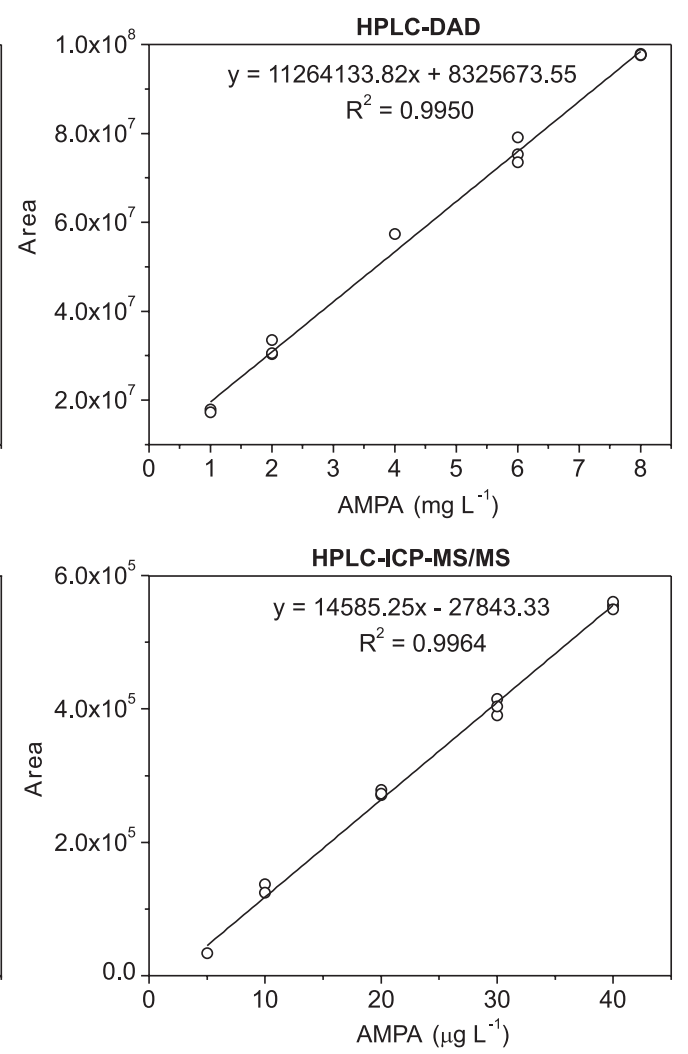

Figure 3. Calibration curves of glyphosate (GLY) and AMPA by HPLC-ICP-MS/MS and HPLC-DAD in the studied concentration ranges, with respective equations and determination coefficients $\left(\mathrm{R}^{2}\right)$. 
Table 3. Comparison of the parameters of the chromatographic systems studied in the determination of glyphosate and AMPA in aqueous samples

\begin{tabular}{lcc}
\hline Parameter & HPLC-DAD & HPLC-ICP-MS/MS \\
\hline Analysis time / min & 20 & $<5$ \\
Derivatization step & yes & no \\
Sample preparation time / min & 200 & 30 \\
Selectivity & yes & yes \\
Linearity & yes & yes \\
Limit of detection / $\left(\mu \mathrm{g} \mathrm{L}^{-1}\right)$ & 300 & 8.2 \\
Price & +++ & + \\
\hline
\end{tabular}

Performance scale: $+=$ moderate, $++=$ good, $+++=$ very good, $++++=$ excellent. HPLC-DAD: high performance liquid chromatography with diode array detector; HPLC-ICP-MS/MS: HPLC using inductively coupled plasma with triple quadrupole mass spectrometer.

that reached for aqueous samples $\left(9 \mu \mathrm{g} \mathrm{L}^{-1}\right)^{29}$ and in apples $\left(10 \mu \mathrm{g} \mathrm{kg}^{-1}\right),{ }^{34}$ but other derivatizing agents were employed in these studies such as 4-chloro-3,5-dinitrobenzotrifluoride. However, the limits of detection achieved in our study were lower than the MRLs established by the US EPA.

\section{Sample preparation time}

The sample preparation includes all the time required for preparing the derivatization reagent solutions such as borate buffer at $\mathrm{pH} 9$ and $\mathrm{FMOC}-\mathrm{Cl}$ reagent, the time for the derivatization reaction to occur, and the preparation of the analytical curve with five concentrations in triplicate for both detection systems. In addition, the samples were not simultaneously derivatized to avoid different waiting times after derivatization. Thus, the mean sample preparation time for the HPLC-DAD analysis was $200 \mathrm{~min}$ due to the derivatization step (Table 3). On the other hand, the mean sample preparation time for determining these analytes by HPLC-ICP-MS/MS was $30 \mathrm{~min}$, because this step was not necessary.

The derivatization step was indispensable in determining glyphosate and AMPA by HPLC-DAD (conventional method), because there is no chromophore group in glyphosate or AMPA molecules. Derivatization is not a simple process, even though it confers higher sensitivity and selectivity to the studied compounds. ${ }^{16}$ Several factors could affect the efficiency of the reaction, hindering the analysis of complex matrices because interferents present in these matrices could decrease the reaction performance and the compound quantification. In addition, the applicability of derivatization agents should be evaluated for each matrix, because the reaction developed for one matrix may not work in another with different characteristics, which contributes to the increased cost, sample preparation and analysis time. ${ }^{13}$

The results in this study have shown significant superiority of the HPLC-ICP-MS/MS method for determining glyphosate and AMPA in water, as this method presented a shorter analysis time and absence of the derivatization step. In addition, the specificity of this detector and the shorter number of stages in sample preparation are considerable advantages for using this technique, because it confers reliability to a method. The detection by ${ }^{31} \mathrm{P}$ atom also confers more sensitivity and selectivity at the elemental analysis level, making it possible to obtain smaller LODs than in HPLC-DAD.

Although HPLC-ICP-MS/MS was superior to HPLC-DAD in almost all analytical parameters evaluated in this study, it is important to highlight that the acquisition and maintenance value of HPLC-ICP-MS/MS was much higher than HPLC-DAD, mainly in the consumption of argon gas. Therefore, the choice of the analytical strategy to quantify these compounds should also take this parameter into account.

\section{Real samples}

After comparing the two methodologies, HPLC-ICP-MS/MS was used to analyze seven different water samples. All selected samples were collected near eucalyptus plantations. However, the results showed that glyphosate and AMPA were not detected in any of the analyzed samples. Similar results were found in applying HPLC-ICP-MS to water samples from the Ohio River near downtown ${ }^{23}$ in a polluted water sample by ion chromatography (IC) using inductively coupled plasma mass spectrometry (IC-ICP-MS), ${ }^{21}$ and in real river water samples using capillary electrophoresis (CE) coupled to ICP-MS. ${ }^{35}$

\section{Conclusions}

In this paper, the strategy of directly determining glyphosate and AMPA in aqueous samples by HPLC-ICP-MS/MS was compared to the conventional method by HPLC-DAD. The method using HPLC-ICP-MS/MS was faster than the conventional method because it does not require the derivatization step of the target analytes before instrumental analysis. In addition, the limit of instrumental detection was about 36.5 times lower than the limit reached by the diode array detector without previous derivatization with FMOC-Cl. The results for real samples did not detect those analytes in the studied water samples. 


\section{Supplementary Information}

Supplementary data are available free of charge at http://jbcs.sbq.org.br as a PDF file.

\section{Acknowledgments}

The authors thank the Fundação de Amparo à Pesquisa do Estado de Minas Gerais (FAPEMIG) (APQ-01429-16) for the financial support. The authors are also grateful to the Conselho Nacional de Desenvolvimento Científico e Tecnológico (CNPq) (420637/2016-8 and 307381/2015-3) for the financial support from research fellowships, the Coordenação de Aperfeiçoamento de Pessoal de Nível Superior (CAPES) and the Universidade Federal de Minas Gerais (UFMG) for the infrastructure provided.

\section{References}

1. Botero-Coy, A. M.; Ibáñez, M.; Sancho, J. V.; Hernández, F.; J. Chromatogr. A 2013, 1292, 132.

2. Marek, L. J.; Koskinen, W. C.; Pest Manage. Sci. 2014, 70, 1158 .

3. International Agency for Research on Cancer (IARC); Evaluation of Carcinogenic Risks to Humans: Some Organophosphate Insecticides and Herbicides, vol.112; IARC: Lyon, France, 2015.

4. Hanke, I.; Singer, H.; Hollender, J.; Anal. Bioanal. Chem. 2008, $391,2265$.

5. Wang, S.; Liu, B.; Yuan, D.; Ma, J.; Talanta 2016, 161, 700.

6. Pinto, E.; Soares, A. G.; Ferreira, I. M. P. L. V. O.; Anal. Methods 2018, 10, 554 .

7. Demonte, L. D.; Michlig, N.; Gaggiotti, M.; Adam, C.; Beldoménico, G. H. R.; Repetti, M. R.; Sci. Total Environ. 2018, 645, 34.

8. Druart, C.; Delhomme, O.; Vaufleury, A.; Ntcho, E.; Millet, M.; Anal. Bioanal. Chem. 2001, 399, 1725.

9. Sun, L.; Kong, D.; Gu, W.; Guo, X.; Tao, W.; Shan, Z.; Wang, Y.; Wang, N.; J. Chromatogr. A 2017, 1502, 8.

10. He, L.; Luo, X.; Jiang, X.; Qu, L.; J. Chromatogr. A 2010, 1217 , 5013.

11. Chen, M.; Cao, Z.; Jiang, Y.; Zhu, Z.; J. Chromatogr. A 2013, $1272,90$.

12. Khrolenko, M. V.; Wieczorek, P. P.; J. Chromatogr. A 2005, 1093, 111.

13. Koskinen, W. C.; Marek, L. J.; Hall, K. E.; Pest Manage. Sci. 2016, 72, 423.
14. Schrübbers, L. C.; Masís-Mora, M.; Rojas, E. C.; Valverde, B. E.; Christensen, J. H.; Cedergreen, N.; Talanta 2016, 146, 609.

15. Oulkar, D. P.; Hingmire, S.; Goon, A.; Jadhav, M.; Ugare, B.; Thekkumpurath, A. S.; Banerjee, K.; J. AOAC Int. 2017, 100, 631.

16. Arkan, T.; Molnár-Perl, I.; Microchem. J. 2015, 121, 99.

17. United States Environmental Protection Agency (US EPA); EPA 816-F-09-004, National Primary Drinking Water Regulation; Washington D.C., United States of America, 2009.

18. International Association of Environmental Analytical Chemistry (IAEAC); Sample Handling of Pesticides in Water; Active: Barcelona, 1994.

19. Chen, Z.; He, W.; Beer, M.; Megharaj, M.; Naidu, R.; Talanta 2009, 78, 852 .

20. Nelson, J.; Hopfer, H.; Silva, F.; Wilbur, S.; Chen, J.; Ozawa, K. S.; Wylie, P. L.; J. Agric. Food Chem. 2015, 63, 4478.

21. Guo, Z. X.; Cai, Q.; Yang, Z.; Rapid Commun. Mass Spectrom. 2007, 21, 1606.

22. Anadón, A.; Martínez-Larrañaga, M. R.; Martínez, M. A.; Castellano, V. J.; Martínez, M.; Martin, M. T.; Nozal, M. J.; Bernal, J. L.; Toxicol. Lett. 2009, 190, 91.

23. Sadi, B. B. M.; Vonderheide, A. P.; Caruso, J. A.; J. Chromatogr. A 2004, 1050, 95.

24. Origin Pro 8, version v8.0725 (B725); OriginLab, Northampton, USA, 2007.

25. Chang, S. Y.; Wei, M.; J. Chin. Chem. Soc. 2005, 52, 785.

26. Corbera, M.; Hidalgo, M.; Salvadó, V.; Microchim. Acta 2006, 153, 203.

27. Peruzzo, P. J.; Porta, A. A.; Ronco, A. E.; Environ. Pollut. 2008, $156,61$.

28. Amelin, V. G.; Bol'Shakov, D. S.; Tretiakov, A. V.; J. Anal. Chem. 2012, 67, 386.

29. Qian, K.; Wang, F.; Li, J.; Cao, Y.; Anal. Chim. Acta 2009, 635, 222.

30. Becker, J. S.; Boulyga, S. F.; Becker, C. P. J.; Buddrus, S.; Przybylski, M.; Anal. Bioanal. Chem. 2003, 375, 561.

31. Bolea-Fernandez, E.; Balcaen, L.; Resano, M.; Vanhaecke, F.; Anal. Bioanal. Chem. 2015, 407, 919.

32. Lajin, B.; Goessler, W.; Talanta 2019, 196, 357.

33. Kovačevič, M.; Goessler, W.; Mikac, N.; Veber, M.; Anal. Bioanal. Chem. 2005, 383, 145.

34. Qian, K.; Tang, T.; Shi, T.; Li, P.; Li, J.; Cao, Y.; J. Sep. Sci. 2009, 32, 2394.

35. Wuilloud, R. G.; Shah, M.; Kannamkumarath, S. S.; Altamirano, J. C.; Electrophoresis 2005, 26, 1598.

Submitted: March 15, 2019

Published online: July 29, 2019 